\title{
Metagenomic Analysis of Bacterial Diversity on Tomato (Solanum lycopersicum L.) Leaves
}

\author{
V. Sumbula ${ }^{1}{ }^{*}$, P. Sainamole Kurian ${ }^{1}$, D. Girija ${ }^{1}$ and K. Anita Cherian ${ }^{1}$ \\ ${ }^{1}$ Department of Plant Pathology, ${ }^{2}$ Department of Agricultural Microbiology, College of \\ Horticulture, Kerala Agricultural University, Thrissur 680656, Kerala, India \\ *Corresponding author
}

\begin{tabular}{|c|}
\hline Keywords \\
\hline $\begin{array}{l}\text { Illumina platform, } \\
\text { Metagenomics, } \\
\text { Operational } \\
\text { taxonomic units } \\
\text { (OTUs), 16SrRNA, } \\
\text { Tomato }\end{array}$ \\
\hline Article Info \\
\hline $\begin{array}{l}\text { Accepted: } \\
17 \text { September } 2020 \\
\text { Available Online: } \\
10 \text { October } 2020\end{array}$ \\
\hline
\end{tabular}

\section{Introduction}

Microbial communities on or around plants play a major role in plant functioning and vigour. These microbial communities are mainly categorized into three types viz.
Plant leaves are colonized by complex microbial communities, which are adapted to the peculiar habitat on leaf lamina. Although the role and ecology of microorganisms in and on the leaves are only partially understood, leaf microbiota could have a beneficial role in plant growth and health. An amplicon metagenomic approach based on 16SrRNA gene on the MiSeq Illumina platform was used to identify the composition of bacterial communities associated with tomato leaves. A total of 44380 high quality 16s DNA sequences were clustered into 365 operational taxonomic units (OTUs). The results of metagenomomic analysis revealed that, tomato leaf bacterial community mainly comprised five dominant and four rare phyla, among which, Firmicutes (109 OTUs) was the most represented followed by Proteobacteria (95 OTUs), Cyanobacteria (46 OTUs), Bacteriodetes (43 OTUs) and Actinobacteria (16 OTUs). Rare phyla include, Verrucomicrobia (3 OTUs), Acidobacteria (2 OTUs) and Gemmatimonadetes (1 OTUs). Clostridia and Gammaproteobacteria was the most abundant classes of Firmicutes and Proteobacteria respectively. The dominant genera were Geitlerinema (33 OTUs), Pantoea(31 OTUs), Clostridium (26 OTUs) and Acinetobacter (24 OTUs). Besides the presence of common commensal leaf bacteria viz. Pantoea Sphingomonas, and Acinetobacter, the occurrence of Clostridium, a food, animal and human pathogenic genus and Geitlerinema, a fresh and marine water cyanobacteria on tomato leaves is an important observation of the present study. As a whole, this study describes the composition of the bacterial communities of tomato leaves, identifying a variety of genera that could exert multiple effects on growth and health of tomato plants. 
through their roles in bio-accessibility of mineral nutrients, protection against pathogens and release of phytohormones to stimulate plant growth (Park et al., 2008; Mendes et al., 2013). The phyllosphere, which consists of the aerial parts of plants has been less intensively studied than the rhizosphere but has received considerable attention in recent years. Phyllosphere primarily of the set of photosynthetic leaves, is one of the most prevalent microbial habitats on earth. The leaf surface habitat is vast: vegetation modelling gives an estimated global leaf area of $508,630,100 \mathrm{~km}^{2}$, which corresponds to $1,017,260,200 \mathrm{~km}^{2}$ of upper and lower leaf surface, an area approximately twice as great as the land surface (Bulgarelli et al., 2013).

The interest in phyllosphere microbiology was initially driven by investigations into plant pathogens, but most phyllospherecolonizing microorganisms live as commensals and/or mutualistic symbionts on their host plants (Lindowand Brandl, 2003; Vorholt, 2012). Phyllosphere communities are involved in functional processes as large in scale as the carbon cycle, nitrogen fixation, and degradation of organic pollutants and pesticide residues (Müllerand Ruppel, 2014). They are also thought to be relevant for plant development and health as biofertilizers, phytostimulators and biopesticides to protect against invading pathogens. Furthermore, interactions in the phyllosphere zone determine the extent to which human pathogens are able to colonize and survive on plant tissues, an area of increasing importance with the rise in cases of human disease associated with consumption of fresh salad, fruit and vegetable produce (Whipps et al., 2008). The third category is the endophytes; microorganisms which can colonize plants internally with- out any apparent adverse effects on the host (Gaiero et al., 2013). There is a growing interest in endophytic microbes on account of their potential use in plant growth promotion, antagonistic effect on pests and pathogens, alleviation of abiotic stress and in phytoremediation (Ryan et al., 2008; Kurian, 2011; Mercado-Blanco and Lugtenberg, 2014). Being internal colonizers, they could provide a barrier against the invading pathogens directly or through the production of bio-active compounds.

The importance of bacterial diversity on and in the plants has an emerging field of research enabling to studying the various beneficial properties of phyllospheric and endophytic bacterial community. Even though plant leaves are ecologically and economically important ecosystem that hosts a large and diverse bacterial community, studies regarding microbial diversity on the leaves are limited. Thus, it is crucial to understand the community structure and diversity of phyllosphere and endophytic bacteria on plant leaves. It could further contribute to understanding the significant roles of plant microbiota in supporting their multiple bioactivities. Moreover, it will help to develop a more effective and less environmentally damaging strategies for improving plant health and plant protection. However, a vast majority of microorganisms are known to be non-amenable for cultivation on common media (Lundberg et al., 2012; Thomasand Sekhar, 2014). Recently, the development of culture-independent highthroughput sequencing-based metagenomic analysis has further allowed us to obtain a global view about community structure and diversity of phylloplane and endophytic microbiome residing in plant tissues (Turner et al., 2013). One of the main advantages of metagenomic approaches over culturedependent methods, is the ability to theoretically detect all organisms that possess the targeted barcode gene. This includes, organisms that cannot or are extremely difficult to culture, which represent about 
$99 \%$ of the total estimated microbial diversity as well as rare taxa that are usually missed by culturing techniques (Price et al., 2014). Culture-independent techniques can provide a more specific, replicable and detailed description of microbial diversity (Bulgari et al., 2014).

Tomato (Solanum lycopersicum L.) is a vital solanaceous vegetable crop of global importance grown in an area of 4.8 million hectares (FAO, 2018). Varied climatic adaptability and high nutritive value made the tomato cultivation more popular in the recent years. This crop is well studied in terms of genetics, genomics, and breeding, thus being an excellent model for basic and applied research related to fruit quality, stress tolerance, and other physiologic traits (Panthee and Chen, 2010; Sahu et al., 2012). Diseases are one of the main problems of the tomato industry all over the world, and the susceptibility of tomato to many pathogenic microorganisms leads to an intense use of agrochemicals (Gajanana et al., 2006). Thus, biological control agents have emerged as an alternative approach for the control of tomato diseases. In this way, an increased knowledge of the ecology of bacterial communities associated to tomato plants will contribute to identify potential candidates for biologic control of tomato diseases and plant growth promotion. Therefore, the present study was aimed to assess the bacterial diversity of tomato leaves by metagenomic analysis.

\section{Materials and Methods}

\section{Collection of samples}

Tomato leaves were collected from research fields at College of Horticulture, Thrissur, Kerala (GPS coordinates: 10.5484 N 76.2857 E) by random sampling technique. Collected samples were kept in sterile plastic bags in a thermally insulated container. All samples were immediately placed on ice and transported to the high-tech seed testing laboratory attached to the Department of vegetable Science, College of Horticulture and stored at $-80{ }^{\circ} \mathrm{C}$ prior to processing.

\section{Metagenomic DNA extraction}

The extraction of metagenomic DNA was done with an objective to construct metagenomic libraries of tomato leaf samples. In order to capture both endophytic and epiphytic fungi, the whole leaf tissue was used for DNA extraction. Collected samples were pooled and aseptically ground to a fine powder in liquid nitrogen using autoclaved pestle and mortar. Ground tissue powder aliquots $(100 \mathrm{mg})$ were then stored at $-80{ }^{\circ} \mathrm{C}$. Metagenomics DNA extraction was done by using the DNeasy Plant Mini kit (QIAGEN, Dusseldorf, Germany). Samples were first mechanically disrupted and then chemically lysed. RNA is removed by RNAse digestion during lysis. Cell debris, precipitated proteins, and polysaccharides were removed and the sample was homogenized by centrifugation through a QIAshredder spin column. Buffering conditions were adjusted and the lysate was loaded onto the DNEasy Plant Mini spin column. During a brief spin, DNA selectively binds to the silica membrane while contaminants pass through. Remaining contaminants and enzyme inhibitors were removed in one or two efficient wash steps. The DNA was finally eluted using elution buffers to obtain $100 \mu \mathrm{l}$ end product. The standard protocol provided in the user manual was followed to obtain the best result.

\section{Qualitative and quantitative analysis of gDNA}

Quality of gDNA was checked on $1 \%$ agarose gel (loaded $5 \mu \mathrm{l}$ ) for the presence of single intact band. The gel was run at $110 \mathrm{~V}$ for 30 mins. $1 \mu$ of each sample was loaded in 
Nanodrop 8000 for determining A260/280 ratio. The DNA was quantified using Qubit dsDNA HS Assay kit (Life Technologies). 1 $\mu \mathrm{l}$ of each sample was used for determining concentration using Qubit ${ }^{2}$ 2.0 Fluorometer.

\section{Preparation of libraries for 2 x 250 bp Run Chemistry}

The amplicon library was prepared using Nextera XT Index Kit (Illumina inc.) as per the 16S Metagenomic Sequencing Library preparation protocol (Part \# 15044223 Rev. B). Primers for the amplification of the V3V4 hyper-variable region (V3-Forward 5' CCTACGGGNBGCASCAG 3' and V4Reverse 5' GACTACNVGGGTATC TAATCC 3') of $16 \mathrm{~S}$ rDNA gene of bacteria were designed in Xcelris NGS Bioinformatics Lab. These primers were synthesized in XcelrisPrimeX facility.

The amplicon with the Illumina adaptors were amplified by using i5 and i7 primers that add multiplexing index sequences as well as common adapters required for cluster generation (P5 and P7) as per the standard Illumina protocol. The amplicon libraries were purified by $1 \mathrm{X}$ AMpureXP beads, checked on Agilent DNA1000 chip on Bioanalyzer 2100 and quantified by Qubit Fluorometer 2.0 using Qubit dsDNA HS Assay kit (Life Technologies).

\section{Cluster generation and sequencing}

After obtaining the Qubit concentration for the library and the mean peak size from bioanalyser profile, library was loaded onto Illumina platform at appropriate concentration (10-20pM) for cluster generation and sequencing. Paired-end sequencing allowed the template fragments to be sequenced in both the forward and reverse directions on Illumina platform. The kit reagents were used in binding of samples to complementary adapter oligos on paired-end flow cell. The adapters were designed to allow selective cleavage of the forward strands after resynthesis of the reverse strand during sequencing. The copied reverse strand was then used to sequence from the opposite end of the fragment.

\section{Bioinformatics analysis and data processing}

Sequence analysis was carried out using the quantitative insights into microbial ecology (QIIME) pipeline (Caporaso et al., 2010). For sequence filtering, reads shorter than 200 bases or longer than 1,000 bases were discarded, sequences with homopolymer runs longer than six bases or more than six ambiguous bases were also discarded, whereas one barcode correction and two primer mismatches were accepted. Chimeras were removed using the UCHIME program $^{23}$ according to the USEARCH pipeline. Operational taxonomic units (OTU) were determined using the UCLUST algorithm (Edgar, 2010) at 97\% sequence similarity. The taxonomy assignment of the bacterial OTU was carried out using the Naïve Bayesian RDP classifier with a minimum confidence of 0.8 (Wang et al., 2007)against the Greengenes database downloaded from October 2012 (McDonald et al., 2012). Singleton OTU was removed for statistical analysis. Demultiplexed raw sequence data were deposited in the Sequence Read Archive (http://www.ncbi.nlm. nih.gov/sra) under the accession number SRR10698108.

\section{Results and Discussion}

The libraries were prepared from the sample after amplifying V3-V4 region $16 \mathrm{~S}$ segment. The $16 \mathrm{~S}$ library mean size was $573 \mathrm{bp}$. The library was sequenced using the Illumina $2 \mathrm{x}$ 250 bp sequencing chemistry to generate $\sim 150 \mathrm{Mb}$ of data per library. The next 
generation sequencing was performed using 2x250 PE chemistry on the Illumina platform. Paired end sequence assembly was carried out for data generated using FLASH (Parameter Minimum overlap of 10 bases) assembler. FLASH (Fast Length Adjustment of Short reads) is a very fast and accurate software tool to merge paired-end reads from nextgeneration sequencing experiments. After the sequence assembly 458124 reads of bacteria were obtained and chimeric sequences were removed from these. Chimeras are DNA sequences composed of DNA from two or more microbial species. They are artefacts made during the PCR process. Hence, after filtering out these chimeric sequences, 443840 reads of bacteria were obtained. These good quality sequences from the samples were clustered into Operational Taxonomic Units (OTUs) based on their sequence similarity. OTUs are clusters of sequences, frequently intended to represent some degree of taxonomic relatedness, each resulting cluster is typically representing a same species. So, in the present study 1715 OTUs were picked up for bacteria and the total number of OTUs obtained after the removal of singletons was 709 . As taxonomic assignment showed presence of large amount of chloroplast and mitochondrial contamination, they were removed from the assignment file before plotting the taxonomic charts and diversity calculation. So, the actual OTUs after the removal of contaminants was 365 (Table 1). Hence, after all the quality evaluations, a total of 443840 high quality sequences were recovered and assigned to 365 OTUs.

To confirm the accuracy of QIIME taxonomic assignments, rarefaction analysis was performed and it indicated that the sequencing depth had been saturated for the sample (Fig. $1)$. This curve is a plot of the number of species as a function of the number of sequences in a sample. The vertical axis displays the diversity of the community, while the horizontal axis displays the number of sequences considered in the diversity calculation.

Alpha diversity summarizes the diversity of organisms in a sample with a single number using different indices viz. Chao 1 estimator, Shannon index and Simpson's diversity index in a habitat/sample. In the present study Chao 1 estimator, Shannon index and Simpson's diversity index were 223, 4.12 and 0.97 respectively. These indices revealed that more than 50 per cent of the estimated bacterial richness was covered by the sequencing effort. The first definition of alpha diversity was proposed by Whittaker (1960) and is referred to as "The richness in species of a particular stand or community, or a given stratum or group of organisms in a stand". Alpha diversity is determined as the diversity within a single sample or set of replications. Chao 1 estimator index includes the observed number of species more specifically the number species singletons (species observed once) and doubletons (species observed twice). Shannon's index (H') measures for both abundance and evenness of the species present in sample. The Simpson index allows estimation the odds that two individual microorganisms sampled at random will belong to the same OTU.

Based on the metagenomic analysis of the complete $16 \mathrm{~S}$ data set, tomato leaf bacteria were represented by total nine phyla include, five dominant phyla and four rare phyla (Fig. 2). Among different phyla identified, members of the phylum Fermicutes were predominant, collectively accounting 109 OTUs. It was followed by phylum Proteobacteria (95), unclassified (50), Cyanobacteria (46), Bacteriodetes (43) and Actinobacteria (16). Rare phyla include Verrucomicrobia (3), Acidobacteria (2) and Gemmatimonadetes (1). Predominance of Fermicutes, Proteobacteria and Actinobacteria as phyllosphere communities 
of different agricultural crops (e.g. wheat, rice, apple, lettuce, and spinach) and naturally growing plants/trees had been reported by several researchers, although the proportions of individual taxa can vary depending on plant species and phenotype, geographical location, time of year, and human intervention (Redford et al., 2010; Lopez-Velasco et al., 2011; Knief et al., 2012).

Population of Fermicutesin the sample is mainly grouped into four orders viz. Bacillales, Lactobacillales, Clostridiales and Selenomonadales (Table 2). Among these, members of Clostridiales were predominant sharing 82 OTUs, and followed by those of Bacillales (7 OTUs), Selenomonadales (7 OTUs) and Lactobacillales (2 OTUs). Order Clostridiales majorly composed of the generaClostridium (26 OTUs), Eubacterium (15 OTUs), Faecalibacterium (15 OTUs), Blautia (11 OTUs), Ruminococcus (10 OTUs) and Roseburia (5 OTUs). The well documented habitat of genus Clostridium is soil, water and decaying organic matter and most of them are capable to survive as saprophytes. But recent studies reveal plants as hosts for epi- or endophytic colonization of Clostridium suggesting possibility of plants as alternate host for major human and animal bacterial pathogens (Kirzinger et al., 2011; Timmers et al., 2012; Zeiller et al., 2015). Pyrosequencing of grapevine leaf microbial communities shows that, most OTUs from grapevine leaves were assigned to genera Clostridium and Staphylococcus (Yousaf et al., 2014). Similarly, Ercolani (2017) reported occurrence and persistence of culturable Clostridium spp. on the leaves of horticultural plants such as basil, celery, lettuce and tomato. In the present study, most abundant class in the phylum Proteobacteria was Gammaproteobacteria (65 OTUs) followed by Alphaproteobacteria (15 OTUs),
Betaproteobacteria (3 OTUs) and Deltaproteobacteria (2 OTUs) (Fig. 2). The genera Pantoea, Acinetobacter and Sphingomonas were predominant in phylum Proteobacteria. The phylum Proteobacteria comprises several species that promote plant growth and also act as biologic control agents of different diseases. A study of bacteria associated to tomato leaves revealed the presence of Proteobacteria both in greenhouse and field-grown plants (Enya et al., 2007) and also identified Bacillus (phylum Firmicutes) and Pantoea (phylum Proteobacteria) strains with strong antifungal activity against tomato pathogens such as Botrytis cinerea and Alternaria solani. Some Pantoea species can produce $N$-acyl-homoserine lactone (AHL) and the plant-growth hormone indole-3-acetic acid (IAA)(Enya et al., 2007), fix nitrogen from the atmosphere (Loiret et al., 2004) and establish quorum sensing systems on leaves, which makes them possible to suppress pathogens on leaves (Frances et al., 2006; Pusey et al., 2011). Sphingomonas spp. is a Gram-negative, rod-shaped aerobic bacterium that is a highly competitive plant leaf colonizer. In a series of experiments, researchers demonstrated that the leaf colonising bacterium Sphingomonas spp. could protect plants against the leafpathogenic Pseudomonas syringae through substrate competition (Innerebner et al., 2011). Carbon partitioning plays an important role for Sphingomonas spp. to be effective antagonists in the phyllosphere (Delmotte et al., 2009). Similarly, some strains of Acinetobacter play an important role in plant growth promotion, as certain strains of this genus are known to be involved in phytostimulation based on the production of plant-growth-promoting hormones, solubilization of phosphate and production of siderophores (Sarode et al., 2009; Sachdev et al., 2010) (Fig. 3). 
Table.1 Reads statistics of $16 \mathrm{~S}$ metagenome data

\begin{tabular}{|c|c|c|c|c|c|c|c|}
\hline $\begin{array}{c}\text { PE } \\
\text { Reads }\end{array}$ & $\begin{array}{c}\text { Total } \\
\text { Reads } \\
(\mathbf{R} 1+\mathbf{R})\end{array}$ & $\begin{array}{c}\text { Flash } \\
\text { Reads }\end{array}$ & $\begin{array}{c}\text { Chimeric } \\
\text { Sequences }\end{array}$ & $\begin{array}{c}\text { Pre- } \\
\text { processed } \\
\text { Reads } \\
\text { (Non- } \\
\text { chimeric } \\
\text { sequences) }\end{array}$ & $\begin{array}{c}\text { Total } \\
\text { OTUs }\end{array}$ & $\begin{array}{c}\text { Total } \\
\text { OTUs } \\
\text { After } \\
\text { Singleton } \\
\text { Removal }\end{array}$ & $\begin{array}{c}\text { Total OTUs } \\
\text { after } \\
\text { contamination } \\
\text { removal }\end{array}$ \\
\hline $\mathbf{4 6 4 5 1 9}$ & 929038 & 458124 & 14284 & 443840 & 1715 & 709 & 365 \\
\hline
\end{tabular}

Table.2 Genus-level taxonomic assemblage of bacterial diversity from predominant phyla

\begin{tabular}{|c|c|c|c|c|}
\hline Phylum & Class & Order & Family & Genus \\
\hline \multirow[t]{2}{*}{ Actinobacteria } & \multirow[t]{2}{*}{ Actinobacteria } & Bifidobacteriales & Bifidobacteriaceae & Bifidobacterium(3) \\
\hline & & Coriobacteriales & Coriobacteriaceae & Collinsella (8) \\
\hline \multirow[t]{3}{*}{ Bacteroidetes } & \multirow[t]{3}{*}{ Bacteroidia } & \multirow[t]{3}{*}{ Bacteroidales } & Bacteroidaceae & Bacteroides (21) \\
\hline & & & Prevotellaceae & Prevotella (8) \\
\hline & & & Rikenellaceae & Alistipes (6) \\
\hline \multirow{3}{*}{ Cyanobacteria } & \multirow{3}{*}{$\begin{array}{l}\text { unclassified (derived } \\
\text { from Cyanobacteria) }\end{array}$} & Chroococcales & Unclassified & Cyanothece (2) \\
\hline & & Nostocales & Nostocaceae & Aphanizomenon (3) \\
\hline & & Oscillatoriales & Unclassified & Geitlerinema (33) \\
\hline \multirow[t]{11}{*}{ Firmicutes } & \multirow[t]{3}{*}{ Bacilli } & \multirow[t]{2}{*}{ Bacillales } & Bacillaceae & Bacillus (5) \\
\hline & & & Paenibacillaceae & Brevibacillus (2) \\
\hline & & Lactobacillales & Lactobacillaceae & Lactobacillus (2) \\
\hline & \multirow[t]{6}{*}{ Clostridia } & \multirow[t]{6}{*}{ Clostridiales } & Clostridiaceae & Clostridium (26) \\
\hline & & & Eubacteriaceae & Eubacterium (15) \\
\hline & & & Lachnospiraceae & Roseburia (5) \\
\hline & & & Ruminococcaceae & Faecalibacterium (15) \\
\hline & & & & Ruminococcus(10) \\
\hline & & & Unclassified & Blautia (11) \\
\hline & \multirow[t]{2}{*}{ Negativicutes } & \multirow[t]{2}{*}{ Selenomonadales } & Acidaminococcaceae & Acidaminococcus(4) \\
\hline & & & Veillonellaceae & Dialister(3) \\
\hline \multirow[t]{8}{*}{ Proteobacteria } & Alphaproteobacteria & Sphingomonadales & Sphingomonadaceae & Sphingomonas (15) \\
\hline & Betaproteobacteria & Unclassified & Unclassified & Unclassified (3) \\
\hline & Deltaproteobacteria & Unclassified & Unclassified & Unclassified (2) \\
\hline & \multirow[t]{5}{*}{ Gammaproteobacteria } & Enterobacteriales & Enterobacteriaceae & Pantoea(31) \\
\hline & & Pseudomonadales & Moraxellaceae & Acinetobacter(24) \\
\hline & & Vibrionales & Vibrionaceae & Vibrio (5) \\
\hline & & Xanthomonadales & Xanthomonadaceae & Stenotrophomonas (3) \\
\hline & & $\begin{array}{l}\text { unclassified (derived } \\
\text { from } \\
\text { Gammaproteobacteria) }\end{array}$ & $\begin{array}{l}\text { unclassified (derived } \\
\text { from } \\
\text { Gammaproteobacteria) }\end{array}$ & $\begin{array}{l}\text { unclassified (derived } \\
\text { from } \\
\text { Gammaproteobacteria) } \\
\text { (2) }\end{array}$ \\
\hline $\begin{array}{c}\text { Unclassified } \\
\text { (derived from } \\
\text { Bacteria) }\end{array}$ & Unclassified & Unclassified & Unclassified & Unclassified (50) \\
\hline
\end{tabular}


Fig.1 Rarefaction curve for bacterial OTUs, clustering at 97\% sequence similarity

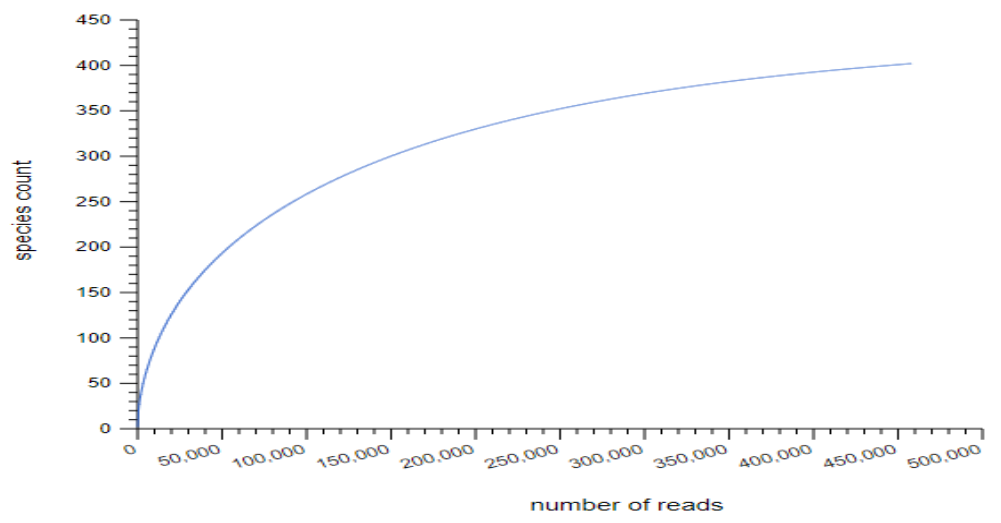

Fig.2 Operational taxonomic units of different phyla

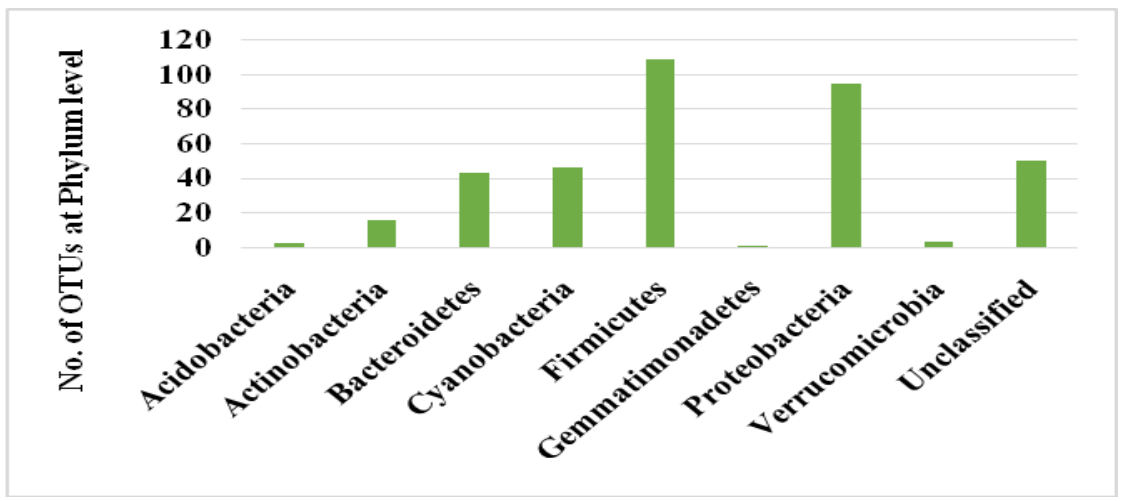

Fig.3 Bacterial classification using RDP Classifier at 97\% identity shown at class level

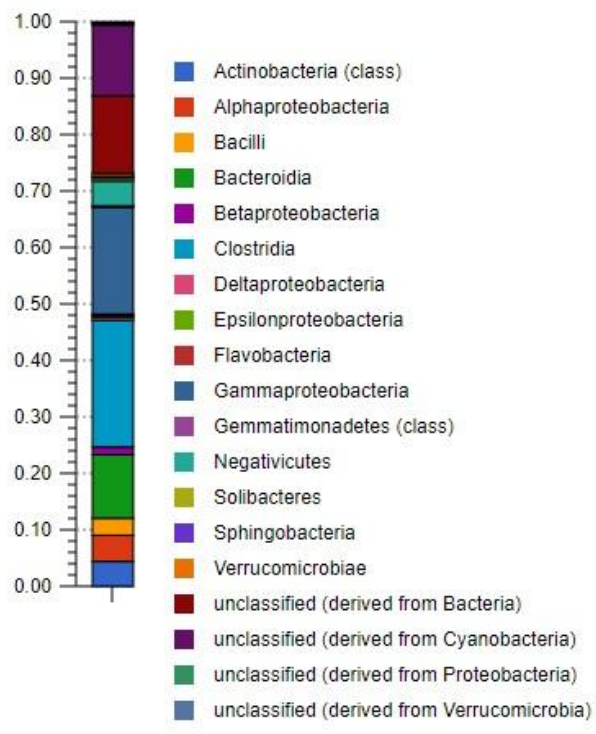


Members of order oscillatoriales were found to be abundant under phylum Cyanobacteria with Geitlerinema as abundant genus. To the best of the authors' knowledge this is the first report of presence of Geitlerinemaon tomato leaves. Genus Geitlerinema is filamentous cyanobacteria commonly inhabiting fresh and marine water. However, different studies have shown increased occurrence of these bacteria in recent decades due to climate change. The filaments and other propagules are being transferred over long distances by air current and wind leading to distribution of these organisms from their source area to other (Broady, 2005 and Pearce et al., 2009). There are reports showing the presence of Geitlerinemain air samples (Genitsaris et al., 2011) and as epiphytes on rice (Zeinab et al., 2013)and mangrove (Alvarenga et al., 2015). Table 1 shows that, the genus Bacteroides and Collinsella dominate among the phyla Bacteroidetes and Actinobacteria respectively. Bacteria belonging to Acidobacteria, Gemmatimonadetes and Verrucomicrobia were less abundant and less diverse on tomato leaves.

In conclusion, the results of the present study indicate that, leaves of tomato plants support a large diversity of bacteria with an abundance of bacterial population from phyla Fermicutes and Proteobacteria. The dominant bacterial genera on the tomato leaves were Geitlerinema (33 OTUs), Pantoea (31 OTUs), Clostridium (26 OTUs) and Acinetobacter (24 OTUs). The presence of commensal bacterial genera viz. Pantoea, Sphingomonas, Acinetobacter are considered as beneficial to the plant, since they have important role in pathogen exclusion, plant growth and productivity. Even though, presence of Clostridial species on tomato leaves was unexpected outcome of present study it certainly merits greater attention because its spores can be added to the tomato fruits leading to contamination of food. Occurrence
Geitlerinema on tomato leaves is another important observation of present study. Moreover, it is important to note that, the unclassified taxa were found to be abundant in the sample and this might indicate that the majority of the leaf bacteria are undiscovered and the activity of these bacteria is still unknown. Regardless of the ratio, the composition of bacterial taxa as whole might play a huge role in mediating the health of tomato plants.

\section{Acknowledgement}

The present work was supported by Kerala Agricultural University, College of Horticulture, Vellanikkara, Thrissur by giving infrastructure and technical facilities.

\section{References}

Alvarenga, D.O., Rigonato, J. and Branco, L.H.Z. 2015. Cyanobacteria in mangrove ecosystems. Biodivers. Conserv. 24: 799-817.

Broady, P.A. 2005. Diversity, distribution and dispersal of Antarctic terrestrial algae. Biodivers. Conserv. 5:1307-1335.

Bulgarelli, D., Schlaeppi, K., Spaepen, S., Themaat, E.V.L. and Schulze-Lefert, P. 2013. Structure and functions of the bacterial microbiota of plants. Annu. Rev. Plant Biol. 64:807-838.

Bulgari, D., Casati, P., Quaglino, F. and Bianco, P.A. 2014. Endophytic bacterial community of grapevine leaves influenced by sampling date and phytoplasma infection process. $B M C$ Microbiol. 14:1-11.

Caporaso, J.G., Kuczynski, J., Stombaugh, J., Bittinger, K. 2010. QIIME allows analysis of high-throughput community sequencing data. Nat. Methods.7:335336.

Delmotte, N., Knief, C., Chaffron, S., Innerebner, G. and Roschitzki B. 2009. 
Community proteogenomics reveals insights into the physiology of phyllosphere bacteria. Proc Natl Acad Sci USA.106:16428-16433.

Edgar, R.C.2010. Search and clustering orders of magnitude faster than BLAST. Bioinformatics, 26:2460-2461.

Enya, J., Shinohara, H., Yoshida, S., Tsukiboshi, T., Negishi, H., Suyama, K. and Tsushima, S. 2007. Culturable leafassociated bacteria on tomato plants and their potential as biological control agents. Microb.Ecol., 53: 524-536.

Ercolani, G.L. 2017. Occurrence and persistence of culturable clostridial spores on the leaves of horticultural plants. J. Appl. Microbiol. 82: 137-140.

Food and Agriculture Organization Corporate Statistical Database. 2018. Available from: http: //en. Wikipedia .org /wiki /Food_and_Agriculture_Organization_ Corporate_Statistical__Database.

Frances, A., Bonaterra, M.C., Moreno, J., Cabrefiga, E. and Badosa, E. 2006. Pathogen aggressiveness and postharvest biocontrol efficiency in Pantoea agglomerans. Postharvest Biol.Tec. 39:299-307.

Gaiero, J.R., McCall, C.A., Thompson, K.A., Day, N.J., Best, A.S. and Dunfield, K.E. 2013. Inside the root microbiome: bacterial root endophytes and plant growth promotion. Am.J.Bot.100:17381750.

Gajanana, T.M., Moorthy, K.P.N., Anupama, H.L., Raghunatha, R. and Kumar, G.T.P. 2006. Integrated pest anddisease management in tomato: an economic analysis. Agric. Econ. Res. Rev.19: 269280.

Genitsaris, S., Kormas, K., Moustaka, G. and Maria.2011. Airborne algae and cyanobacteria: Occurrence and related health effects. Frontiers in bioscience (Elite edition). 3:772-787.

Innerebner, G., Knief, C., Vorholt, A. 2011.
Protection of Arabidopsisthaliana against Leaf-Pathogenic Pseudomonas syringae by Sphingomonas Strains in a Controlled Model System. Appl. Environ. Microbiol. 77 (10): 32023210.

Kirzinger, M. W. B., Nadarasah, G. and Stavrinides, J.2011. Insights into crosskingdom plant pathogenic bacteria. Genes, 2: 980-997.

Knief, C., Delmotte, N., Chaffron, S., Stark, M., Innerebner, G., Wassmann, R., von Mering, C. and Vorholt, J.A. 2012. Metaproteogenomic analysis of microbial communities in the phyllosphere and rhizosphere of rice. ISME. J.6:1378-1390.

Kurian, S.P. 2011. Endophytic microorganism mediated systemic resistance in cocoa against Phytophthora palmivora(Butler) Butler. PhD thesis, Kerala Agricultural University, Thrissur, 197p.

Lindow, S.E. and Brandl, M.T. 2003. Microbiology of the phyllosphere. Appl. Environ. Microbiol. 69:1875-1883.

Loiret, F.G., Ortega, E., Kleiner, D., Ortega- Rodés, P and Rodés, R. 2004. A putative newendophytic nitrogen- fixing bacterium Pantoea sp. from sugarcane. J. Appl. Microbiol. 97(3):504-511.

Lopez-Velasco, G., Welbaum, G.E., Boyer, R.R., Mane, S.P. and Ponder, M.A. 2011. Changes in spinach phylloepiphytic bacteria communities following minimal processing and refrigerated storage described using pyrosequencing of $16 \mathrm{~S}$ rRNA amplicons. J. Appl. Microbiol., 110: 1203-1214.

Lundberg, D.S., Lebeis, S. L., Paredes, S.H. and Lundberg, D. S. 2012. Defining the core Arabidopsis thaliana root microbiome. Nature, 488: 86-90.

McDonald, D., Price, M.N., Goodrich, J., Nawrocki, E.P., DeSantis, T.Z., Probst, 
A., Andersen, G.L., Knight, R. and Hugenholtz, P. 2012. An improved Greengenes taxonomy with explicit ranks for ecological and evolutionary analyses of bacteria and archaea. ISME J.6:610-618.

Mendes, R., Garbeva, P. and Raaijmakers, J.M. 2013. The rhizosphere microbiome: significance of plant beneficial, plant pathogenic, and human pathogenic microorganisms. FEMS Microbiol Rev. 37(5): 634-63.

Mercado-Blanco, J. and Lugtenberg, B.J.J. 2014. Biotechnological applications of bacterial Curr.Biotechnol.3: 60-75.

endophytes.

Müller, T. and Ruppel, S. 2014. Progress in cultivation-independent phyllosphere microbiology. FEMS Microbiol. Ecol.87:2-17.

Panthee, D. and Chen, F.2010. Genomics of fungal disease resistance in tomato. Curr. Genomics, 11: 30-39.

Park, J.H., Kim, R., Aslam, Z., Jeon, C.O. and Chung, Y.R. 2008. Lysobacter capsica sp. nov., with antimicrobial activity, isolated from the rhizosphere of pepper, and emended description of the genus Lysobacter. Int. J. Syst. Evol. Microbiol., 58:387-392.

Pearce, D.A., Bridge, P.D., Hughes, K.A., Sattler, B., Psenner, R. and Russell, N.J. 2009. Microorganisms in the atmosphere over Antarctica. FEMS. Microbiol. Ecol., 69:143-157.

Price, L.B., Liu, C.M., Melendez, J.H., Frankel, Y.M., Engelthaler, D., Aziz, M.2014. Community analysis of chronic wound bacteria using 16S rRNA genebased pyrosequencing: impact of diabetes and antibiotics on chronic wound microbiota. PLoS ONE.4: e6462.

Pusey, P., Stockwell, Virginia, R., Catherine, S., and Duffy, B. 2011. Antibiosis Activity of Pantoea agglomerans
Biocontrol Strain E325 Against Erwinia amylovora on Apple Flower Stigmas. Phytopathology. 101:1234-1241.

Redford, A.J, Bowers, R.M., Knight, R., Linhart, Y., and Fierer, N. 2010. The ecology of the phyllosphere: geographic and phylogenetic variability in the distribution of bacteria on tree leaves. Environ. Microbiol.12: 2885-2893.

Ryan, R.P., Germaine, K., Franks, A., Ryan, D.J. and Dowling, D.N. 2008. Bacterial endophytes: recent development and applications. FEMS Microbiol. Lett.278: $1-9$.

Sachdev, D., Nema, P., Dhakephalkar, P., Zinjarde, S. and Chopade, B.2010. Assessment of 16S rRNA gene based phylogenetic diversity of Acinetobacter community from the rhizosphere of wheat. Microbiol. Res. 165: 627-638.

Sahu, P., Puranik, S., Khan, M and Prasad, M. 2012. Recent advances in tomato functional genomics: utilization of VIGS. Protoplasma.249: 1017-1027.

Sarode, P. D., Rane, M.R., Chaudhari, B.L. and Chincholkar, S.B. 2009. Siderophoregenic Acinetobacter calcoaceticus isolated from wheat rhizosphere with strong PGPR activity. Malaysian J. Microbiol. 5: 6-12.

Thomas, P. and Sekhar, A.C. 2014. Live cell imaging reveals extensive intracel- lular cytoplasmic colonization of banana genotypes by normally non-cultivable endophytic bacteria. $A o B$ Plants.6:plu002.

Timmers, R. A., Rothballer, M., Strik, D. P., Engel, M., Schulz, S. and Schloter M. 2012. Microbial community structure elucidates performance of Glyceria maxima plant microbial fuel cell. Appl. Microbiol. Biotechnol.94: 537-548.

Turner, T. R., James, E.K. and Poole, P.S.2013. The plant microbiome. Genome Biol., 14: 209.

Vorholt, J.A. 2012. Microbial life in the 
phyllosphere. Nat. Rev. Microbiol. 10:828-840.

Wang, Q., Garrity, G.M., Tiedje, J.M. and Cole, J.R. 2007. Naive Bayesian classifier for rapid assignment of rRNA sequences into the new bacterial taxonomy. Appl. Environ. Microbiol. 73:5261-5267.

Whipps, J.M., Hand, P., Pink, D. and Bending, G.D. 2008. Phyllosphere microbiology with special reference to diversity and plant genotype. J. Appl. Microbiol. 105: $1744-1755$.

Whittaker, R.H. 1960. Vegetation of the Siskiyou Mountains, Oregon and California. Ecological Monograph. 30:279-338.

Yousaf, S., Bulgari, D., Bergna, A., Pancher,
M., Quaglino, F., Casati, P. and Campisano, A. 2014. Pyrosequencing detects human and animal pathogenic taxa in the grapevine endosphere. Frontiers in microbiology, 5: 327.

Zeiller, M., Rothballer, M., Iwobi, A. N., Böhnel, H., Gessler, F., Hartmann, A. and Schmid, M. 2015. Systemic colonization of clover (Trifolium repens) by Clostridium botulinum strain 2301. Frontiers in microbiology, 6:1207.

Zeinab, S., Hossein, R. and Mehri, S.H. 2013. Plant growth promoting cyanobacteria and their distribution in terrestrial habitats of Iran. Soil biol.535-547.

\section{How to cite this article:}

Sumbula, V., P. Sainamole Kurian, D. Girija and Anita Cherian, K. 2020. Metagenomic Analysis of Bacterial Diversity on Tomato (Solanum lycopersicum L.) Leaves. Int.J.Curr.Microbiol.App.Sci. 9(10): 2162-2173. doi: https://doi.org/10.20546/ijcmas.2020.910.263 